Editorial

\title{
Acknowledgment to Reviewers of Vaccines in 2021
}

\author{
Vaccines Editorial Office
}

Citation: Vaccines Editorial Office. Acknowledgment to Reviewers of Vaccines in 2021. Vaccines 2022, 10 213. https://doi.org/10.3390/ vaccines10020213

Published: 29 January 2022

Publisher's Note: MDPI stays neutral with regard to jurisdictional claims in published maps and institutional affiliations.

Copyright: (C 2022 by the author. Licensee MDPI, Basel, Switzerland. This article is an open access article distributed under the terms and conditions of the Creative Commons Attribution (CC BY) license (https://creativecommons.org/licenses/by/4.0/).

MDPI AG, St. Alban-Anlage 66, 4052 Basel, Switzerland

Rigorous peer-reviews are the basis of high-quality academic publishing. Thanks to the great efforts of our reviewers, Vaccines was able to maintain its standards for the high quality of its published papers. Thanks to the contribution of our reviewers, in 2021, the median time to first decision was 17 days and the median time to publication was 39 days. The editors would like to extend their gratitude and recognition to the following reviewers for their precious time and dedication, regardless of whether the papers they reviewed were finally published:

Abate, Andrea

Abbate, Jessica L.

Abraham, Rachy

Abravanel, Florence

Abril, Carlos

Acheson, Ashley

Acquasaliente, Laura

Adadi, Parise

Adamovicz, Jeffrey J.

Adan, Ana

Adunyah, Samuel E.

Aggarwal, Megha

Agha, Sohail

Agmon-Levin, Nancy

Agostini, Simone

Agostinis, Chiara

Aguinaga-Ontoso, Inés

Agyei-Baffour, Peter

Ahad, Abdul

Ahlenstiel, Chantelle

Ahmad, Khurshid

Ahmad, Sajjad

Ahmadian, Gholamreza

Aisa, Rosa

Ajejas, Julia

Akbar, Sheikh Mohammad Fazle

Akbulut, Sami

Alaimo, Alessandro

Albendín-García, Luis

Alberola, Juan

Albrecht, David

Alcolea, Pedro J.

Alderson, Mark

Alexander, H. Denis
Alfano, Gaetano Ali, Hayssam M.

Alkan, Michael

Allain, Jean-Pierre

Al-Lami, Faris

Allegaert, Karel

Allington, Daniel

Al-Maharik, Nawaf

Almazan, Elbert

Alsaggaf, Rotana

Altieri, Marta

Altmann, Danny

Amarilla, Alberto A.

Amarowicz, Ryszard

Amodio, Emanuele

Andonov, Anton

Andréani, Julien

André-Fontaine, Genevieve

Andreoni, Francesca

Andreotti, Giuseppina

Angelillo, Italo

Angelini, Corrado

Angius, Fabrizio

Anisimov, Andrey P.

Ansa, Benjamin

Anticoli, Simona

Antoñanzas, Fernando

Antonyuk, Svetlana

Antunes, Joana

Anwar, Firoz

Aranda, Carlos J.

Aranday-Cortes, Elihu

Arciszewski, Marcin Bartłomiej

Aremu, Olatunde 
Aremu, Taiwo

Arena, Fabio

Ariani, Alarico

Armengot-Carceller, Miguel

Arnason, Gardar

Arora, Arinder K.

Arora, Gunjan

Arukha, Ananta Prasad

Asakawa, Mitsuhiko

Asare, Matt

Asdaq, Syed M.B.

Aseervatham, Jaya

Ashbrook, Alison

Asin, Javier

Attia, Sameh

Attri, Kuldeep

Atzori, Laura

Audet, Jonathan

Axiotis, Evangelos

Ayala, Juan

Aziz, Faisal

Aziz, Khaled

Azuma, Chieko

Azzi, Alberta

Babiuk, Lorne

Babiuk, Shawn

Bachmann, Martin

Bae, SeungJin

Bahmad, Hisham

Bahrs, Christina

Băicuș, Anda

Baicus, Cristian

Bailey, Adam Lee

Baindara, Piyush

Baiu, Dana Carina

Baj, Andreina

Baker, David

Bakrania, Bhavisha

Bala, Saikat

Balaraman, Velmurugan

Balasubramaniam, Muthukumar

Baldanzi, Gianluca

Balieva, Flora N.

Ball, Patrick A.

Balla, Keir M.

Balzani, Agnese

Bamford, Connor

Banadyga, Logan

Banerjee, Kasturi

Bankala, Krishnarjuna

Bankwitz, Dorothea
Bannantine, John P.

Bansal, Anju

Bao, Yongming

Baquero, Monica M.

Bara, Jeffrey

Barata, Pedro

Barbezange, Cyril

Barchitta, Martina

Barrett, Alan

Barroso, Helena

Baršić, Bruno

Bartholomeeusen, Koen

Bartok, Eva

Bartoszek, Agnieszka

Bärwolff, Günter

Bashyal, Santosh

Bastos, Armanda

Basu, Sankar

Basu, Saumik

Batchelor, Paul

Bates, John T.

Batista-Duharte, Alexander

Batra, Himanshu

Becker, Katrin Anne

Behnam, Mira A.M.

Bélanger, Kasandra

Beliakova-Bethell, Nadejda

Beloukas, Apostolos

Belov, George

Beltowski, Jerzy

Benati, Daniela

Bencúrová, Elena

Ben-David, Boaz

Benevene, Paula

Benfield, David A.

Benítez-King, Gloria

Bennardo, Luigi

Bennett, Julie

Benos, Alexis S.

Bentel, Jacqueline M.

Bera, Soumen

Beran, Jiri

Bercovier, Herve

Berezin, Eitan Naaman

Bergh, Øivind

Berkowska, Magdalena A.

Berri, Fatma

Bertau, Martin

Berthelot, Laureline

Bertram, Miranda

Bertzbach, Luca Danilo 
Berzero, Giulia

Bestetti, Reinaldo B.

Bettencourt, Paulo

Bettridge, Judy

Beyi, Ashenafi F.

Bezirtzoglou, Eugenia

Bhagavathula, Akshaya Srikanth

Bhargava, Aditi

Bhattacharya, Anannya

Bhattacharyya, Sanchari

Bhawal, Ujjal Kumar

Bhusetty Nagesh, Prashanth Kumar

Bhutta, Mahmood Fazal

Bianchi, Francesco Paolo

Bianco, Francesco

Bidmos, Fadil

Bielefeldt-Ohmann, Helle

Bikov, Andras

Bins, Adriaan D.

Biondo, Carmelo

Birlea, Marius

Biswas, Sudipta

Blair, Carol

Blanco-Lobo, Pilar

Blasco, Jose Maria

Bleilevens, Christian

Blunt, Matthew

Bobkova, Marina

Bocedi, Alessio

Bock, Claus-Thomas

Bock, Wolfgang

Bogers, Willy M.J.M.

Bogucki, Jacek

Bohannon, Kevin

Böhmer, Merle M.

Bohmwald, Karen

Bommireddy, Ramireddy

Bonay, Marcel

Bongiovanni, Marco

Bonini, Sergio

Boquet, Didier

Borges, Margarida

Borkow, Gadi

Boroń-Kaczmarska, Anna

Bos, Nico

Bose, Rajendran J.C.

Botgros, Radu

Böttcher, Sindy

Bottero, Julie

Bouman, Judith

Bourdon, Emmanuel
Bowen, Deborah

Braack, Malte

Bracco, Enrico

Branca, Jacopo Junio Valerio

Brandenburg, Lars-Ove

Brandt, Sabine

Brieger, Angela

Brochot, Etienne

Broecker, Felix

Brogi, Simone

Brogna, Barbara

Brooks, Benjamin D.

Brown, Catherine M.

Brown, Mark A.

Brown, Richard J.P.

Brugliera, Luigia

Brunton, Lucy

Bruzzese, Dario

Bruzzese, Francesca

Bsisu, Isam

Buchta, Christoph

Buddaseth, Salma

Bugarin, Alejandro

Bukrinsky, Michael

Bungau, Simona

Burckhardt, Christoph

Burge, Russel

Burián, Katalin

Burton, Zachary F.

Buseyne, Florence

Buxton, Samuel

Byadgi, Omkar Vijay

Caballero, Pablo

Cabaret, Jacques

Cabeda, José Manuel

Cadeddu, Chiara

Cai, Shuowei

Cain, Derek W.

Calagna, Gloria

Calina, Daniela

Callendret, Benoit

Calligari, Paolo

Campbell, Nicole K.

Campello, Elena

Campioni, Paolo

Campo, Flaminia

Campus, Guglielmo

Camus-Bouclainville, Christelle

Cantini, Francesca

Canuti, Marta

Caputo, Antonella 
Cara, Andrea

Carbajo-Lozoya, Javier

Cardoso, Fernando

Cardoso, Luís

Careem, Faizal

Carestia, Mariachiara

Carlo, Signorelli

Carmagnola, Daniela

Carmignani, Marco

Carneiro, Luiz

Carnell, George

Carr, Robert A.

Carrasco, Adriano

Carreras, Joaquim

Carvalho, Edmund

Cascone, Giuseppe

Cassimos, Dimitrios

Castaldi, Silvana

Castellazzi, Massimiliano

Castiello, Luciano

Castiglia, Paolo

Castro Esteves, Pedro José

Cattalini, Marco

Cedrone, Fabrizio

Cerón-Carrasco, José P.

Cerqueira, Laura

Chabot, Donald J.

Chackerian, Bryce

Chaganty, Bharat

Chaguza, Chrispin

Chakrabarti, Jayati

Chan, Kwok-Hung

Chan, Martin

Chandrashekar, Abishek

Chao, Yu-Chan

Chappell, Keith

Chatterjee, Sampurna

Chatterjee, Satabdi

Chavez-Calvillo, Gabriela

Chawla-Sarkar, Mamta

Cheloha, Ross

Chen, Chuan

Chen, Chung-Ming

Chen, Gang

Chen, Kuo-Hu

Chen, Lechuang

Chen, Shanquan

Chen, Shicheng

Chenais, Benoit

Cheng, Zishuo

Chesneau, Christophe
Chiancone, Francesco

Chies, José

Chiffi, Daniele

China, Bernard

Chinnici, Gaetano

Chinta, Krishna C.

Chiostri, Marco

Chiozzini, Chiara

Chlichlia, Katerina

Cho, Do-Yeon

Choi, Chang Won

Choi, Hyo Geun

Choi, Seong-O

Chokshi, Aalap

Chong, Roger

Choudhary, Alok

Chow, Franklin W.N.

Chowdhury, Imran Hussain

Chowdhury, Shafiqul

Christodoulides, Myron

Chua, Eng Guan

Chuang, Hung-Yi

Chun, Kwang-Hoon

Chung, Hee-Chun

Çiftçi, Halil İbrahim

Cimmino, Flora

Cingolani, Mariano

Cingoz, Oya

Cipolloni, Luigi

Circella, Elena

Clark, Andrew

Clarke, Penny

Clemens, Mark

Clement, Cristina

Climent, Joan

Cochis, Andrea

Colebunders, Robert

Coleman, Christopher

Colla, Emanuela

Collins, Catherine

Collura, Ada

Colman, Ricki

Comas-Garcia, Andreu

Comas-Garcia, Mauricio

Conn, David Bruce

Conners, Gregory

Console, Lara

Conte, Ferruccio

Conti, Pio

Contu, Paolo

Conway, Brian 
Coombs, Kevin

Coppeta, Luca

Cordero, Oscar

Cortes-Cerisuelo, Miriam

Cosby, Sara Louise

Cosseddu, Gian Mario

Cossu, Davide

Costanza, Massimo

Coudert, Jerome

Criado-Alvarez, Juan José

Crooks, George

Crupi, Mathieu J.F.

Cruz Hervert, Luiz Pablo

Cugliari, Giovanni

Cull, Benjamin

Cusick, Matthew F.

Cuypers, Bart

D'Amelio, Raffaele

D'Apice, Luciana

Da Fonseca, Flavio Guimaraes

Da Silva, Henrique Borges

Dahiya, Satinder

Dal Col, Jessica

Damiani, Daniela

Damiani, Giovanni

Danovaro-Holliday, Carolina

Darrow, Jonathan

Datta, Prasun K.

Davidson, Irit

Davies, Anita A.

Dawen Yu, Esther

Dawood, Mahmoud A.O.

De Alwis, Ruklanthi (Rukie)

De Andrés Calle, Rocío

De Araújo, Tânia Maria

De Bonis, Salvatore

De Bragança Pereira, Carlos Alberto

De Jonge, Marien

De La Maza, Luis M.

de la Torre, Juan C.

De Perio, Marie

De Robertis, Mariangela

De Sanctis, Juan Bautista

De Silva, Pushpamali

De Simone, Salvatore Giovanni

De Vita, Alessandro

De Waele, Jorrit

Deback, Claire

Debela, Ahmed M.

Debin, Tian

Deere, Jesse
Degiannis, Dimitrios

Del Fresno Sánchez, Carlos

Del Mistro, Annarosa

Del Riccio, Marco

Delbue, Serena

Delcea, Camelia

Delhon, Gustavo

Depken, Craig

Deplanque, Dominique

DeStefano, Christin B.

Devasundaram, Santhi

Devaurs, Didier

Devy, Zisman

Dewals, Benjamin

Dhakal, Santosh

Dhungyel, Om

Di Bella, Santina

Di Bona, Danilo

Di Domizio, Jeremy

Di Gennaro, Francesco

Di Giovanni, Pamela

Di Giuseppe, Gabriella

Di Guilmi, Anne-Marie

Di Maria, Emilio

Di Martino, Giuseppe

Di Profio, Federica

Di Stefano, Mariantonietta

Dias, Joana

Díaz-Rosales, Patrícia

Dielschneider, Rebecca

Diemberger, Igor

Diesfeld, Kate

Dijkstra, Frederika

Dimitrov, Kiril

Ding, Lingwen

Dixon, Linda

Dkhar, Hedwin Kitdorlang

Docea, Anca

Dolan, Brian P.

Dolinski, Dariusz

Domenech, Ana

Domingo, Cristina

Domnich, Alexander

Donadu, Matthew

Dondi, Arianna

Dong, Chunhong

Donini, Marcello

Dörr, Marcus

Dory, Daniel

Dos Santos Dias, Lucas

Dosoky, Noura 
Dowd, Kimberly A.

Doytchinova, Irini

Drillien, Robert

Duarte, Ricardo

Dubé, Mathieu

Dubovi, Edward

Ducatez, Mariette

DuCros, Philipp

Duddempudi, Phaneendra K.

Dudhipala, Narendar

Dulebenets, Maxim

Dumache, Raluca

Dumitrescu, Oana

Dunn, John

Duplaga, Mariusz

Duque, Hernando

Dyer, Arthur

Dzimianski, John

Ebensperger, Germán

Ebina, Hirotaka

Eccher, Albino

Eckels, Kenneth H.

Edith Rojas, Anaya

Edwards, Emily S.J.

Ehrhardt, Matthias

Ehtisham-ul-Haque, Syed

Eibl, Martha M.

Eick, Christian

El Hadi, Hamza

Eldridge, Sandy

Elena, Criscuolo

Elguezabal, Natalia

Elharrak, Mehdi

Eljaafari, Assia

Elkrief, Arielle

Emran, Talha Bin

Endo, Makoto

Engelmann, Ilka

Escaffre, Olivier

Escudero-Pérez, Beatriz

Espinosa, Emma

Essani, Karim

Esser, Mark

Esworthy, Robert Steven

Faaberg, Kay

Fabris, Martina

Fadda, Marta

Faita, Francesco

Fallarini, Silvia

Falzone, Luca

Faquim-Mauro, Eliana L.
Fara, Gaetano Maria

Farina, Antonella

Farnos, Omar

Farronato, Marco

Farsang, Attila

Faure-Dupuy, Suzanne

Fedele, Giorgio

Feldman, Charles

Feng, Huapeng

Feng, Ningguo

Fernandes, Elizabeth S.

Fernandes-Pedrosa, Matheus De Freitas

Fernández-Alarcón, Claudia

Fernández-Prados, Juan Sebastián

Fernández-Soto, Pedro

Ferorelli, Davide

Ferraioli, Giovanna

Ferrantelli, Flavia

Ferrara, Pietro

Ferree, Karen

Ferreira, David

Ferreira, Fernando

Fiems, Dieter

Fiester, Steve

Fife-Schaw, Chris

Filippini, Francesco

Filippou, Panagiota S.

Fiore, Maria

Firczuk, Małgorzata

Fischer, Carlo

Fletcher, Nicola F.

Flisiak, Robert

Flora, Gagan

Fol, Marek

Fonseca, Gregory J.

Formenti, Paolo

Forsyth, Donelson R.

Fossum, Caroline

Fossum, Even

Fowke, Keith

Foyle, Leo

Fraguas-Sánchez, Ana Isabel

Fraleoni-Morgera, Alessandro

Fraley, Gregory S.

Franchini, Massimo

Franciosini, Maria

Franco, David

Franzoni, Giulia

Freer, Giulia

Fregeneda-Grandes, Juan-Miguel

Freiberg, Alexander N. 
Freick, Markus

Frigo, Lúcio

Fronteira, Ines

Frossard, Jean-Pierre

Frutos, Roger

Fry, Lindsay

Fthenakis, George

Fujisawa, Takao

Fuller, A. Oveta

Fuller, Frederick J.

Furlini, Giuliano

Furuya, Tetsuya

Gacesa, Ranko

Gaikwad, Hanmant

Gaíllat, Jacques

Gajdács, Márió

Gala, Rikhav P.

Galanis, Alexis

Gałas, Aleksander

Galindo-Villegas, Jorge

Gallegos-Arreola, Martha Patricia

Gallo, Gaetano

Gaman, Mihnea-Alexandru

Gamazo, Carlos

Gamil, Amr

Gan, Lydia

Gan, Samuel Ken-En

Ganju, Aditya

Ganta, Roman

Garcia-Dorival, Isabel

Garcia-Moreno, Francisca

García-Muñoz Rodrigo, Fermin

Garfinkel, David

Garijo Toledo, María Magdalena

Garner, Bianca L.

Gärtner, Barbara

Garufi, Carlo

Gatto, Francesca

Gaudreault, Natasha N.

Gazerani, Parisa

Ge, Baoxue

Gea-Banacloche, Juan Carlos

Gellert, George A.

Genovese, Cristina

Gergely, Lajos

Gerhard, Markus

Gershwin, Laurel

Gessler, Florian

Getti, Giulia T.M.

Ghilardi Lago, João Henrique

Ghodake, Gajanan
Ghosh, Mrinmoy

Ghosh, Prithwi

Giacomelli, Andrea

Giamberardino, Paolo Di

Giammanco, Anna

Gianfredi, Vincenza

Giangaspero, Massimo

Giannella, Luca

Giantsis, Ioannis

Gieryńska, Małgorzata

Gilbert, Amy Turmelle

Gilbert, Leona

Gilmour, Stuart

Gimenez Llort, Lydia

Gimple, Ryan C.

Girard, Marc

Giudici, Paolo

Giungato, Pasquale

Giuseppe, Arcangeli

Gladue, Douglas

Gnat, Sebastian

Gobbi, Giuliana

Godfroid, Jacques

Godman, Brian

Gogoi, Himanshu

Goldman, Alejandra

Gómez Cerezo, Jorge Francisco

Gomez Del Moral, Manuel

Gomez, Carmen Elena

Gomez-Casado, Eduardo

Gómez-Sebastián, Silvia

Gómez-Urquiza, Jose L.

Gonçalves-Pereira, João

Goncharuk, Anatoliy

Gong, Lan

Gongal, Gyanendra

Gonzalez, Gabriel

González, María Eugenia

González-García, Alberto

González-Granado, Luis Ignacio

Gonzalez-Parra, Gilberto

Gonzalez-Rodriguez, Alexandre

Gonzalez-Scarano, Francisco

Goodla, Lavanya

Goodman, Alan G.

Gopal, Radha

Gordon, Stuart

Gori, Davide

Gorini, Francesca

Gouandjika-Vasilache, Ionela

Gould, Ernest A. 
Graham, Melanie L.

Granero-Molina, Jose

Gregorio, Cesare De

Greim, Helmut

Grilli, Roberto

Grinnemo, Karl Henrik

Gris-Oliver, Albert

Grizzi, Fabio

Groff, Joseph

Groschup, Martin

Grose, Charles

Grosse, Scott D.

Grossegesse, Marica

Grossman, Zvi

Grubczak, Kamil

Grubman, Marvin

Grubor-Bauk, Branka

Grześk, Grzegorz

$\mathrm{Gu}$, Haidong

Gudey, Shyam Kumar

Guerriero, Ilaria

Guest, Johnathan D.

Guilherme, Luiza

Guillen-Grima, Francisco

Guimarães, Jorge A.

Gul, Sheraz

Gulla, Krishna

Guo, Huichen

Guo, Sijin

Gutfraind, Alexander

Guthmiller, Jenna J.

Gutierrez-Guerrero, Alejandra

Gutiérrez-Martin, César B.

Haase, Elaine M.

Haeder, Simon F.

Hahn, Alexander

Hai, Rong

Hall, Robyn

Hall, Roy A.

Haller, Bernhard

Hamilton-West, Christopher

Hammerl, Jens André

Hampson, Ian

Han, Dohyun

Han, DongWoon

Han, Jae Yong

Hang, Jun

Hans, Aymeric

Hantz, Sébactien

Harada, Mamoru

Harkiss, Gordon
Harris, Audray K.

Harrod, Robert

Haslwanter, Denise

Hassandarvish, Pouya

Hatmal, Ma'mon M.

Havrdova, Eva

Hawkes, David

Hearn, Michael J.

Heegaard, Peter M. H.

Hefferon, Kathleen L.

Hegde, Bindu

Hegele, Richard G.

Heidari, Mohammad

Heller, E. Dan

Helsloot, Ira

Henderson, Rory

Heneberg, Petr

Hepojoki, Satu

Herchenröder, Ottmar

Hernaez, Bruno

Hernandez, Jesus

Hernández-Breijo, Borja

Hernández-Castro, Rigoberto

Herod, Morgan

Herrera, Guadalupe

Herzberg, Philipp Y.

Hess, Michael L.

Heuft, Hans-Gert

Higson, Edward

Hildt, Eberhard

Hinds, Lyn A.

Hinkula, Jorma

Hoang Vuong, Quan

Hoelzle, Ludwig E.

Hoffmann, Bernd

HogenEsch, Harm

Holbrook, Michael

Honko, Anna

Hooker, Angelo B.

Hooper, Douglas C.

Horai, Yoshiro

Horefti, Elina

Horii, Toshihiro

Horn, Martin

Horobet, Alexandra

Horstman, Klasien

Hort, Krishna

Horton, John

Hostiuc, Mihaela

Hour, Mann-Jen

Hozbor, Daniela 
Hsieh, Ming-Kun

Huang, Hui

Huang, Jian-dong

Huang, Jiansheng

Huang, Junming

Huang, Sheng-Wen

Hulme, John

Hung, Shuen-Iu

Hurley, James

Hussain, Ahmad Fawzi

Hutchinson, Katrina

Iampietro, Mathieu

Iborra, Salvador

Iborra-Egea, Oriol

Ide, Kazuki

Iijima, Norifumi

Ikebuchi, Ryoyo

Ilinykh, Philipp A.

Illing, Patricia T.

Ilyinskii, Petr O.

Imamura, Ryu

Imbimbo, Bruno

Imperatore, Roberta

Innes, Carrie

Inoue, Jun

Iordanskiy, Sergey

Iorio, Ronald M.

Irigoyen-Camacho, María Esther

Irina, Chelysheva

Isaacs, Stuart

Isel, Catherine

Ishigaki, Yasuhito

Islam, Ariful

Ismael, Maria Isabel

Isola, Gaetano

Isola, Miriam

Ita, Kevin

Iturriza-Gomara, Miren

Iwagami, Moritoshi

Iwata, Kentaro

Iwu, Chinwe

Izumida, Mai

Jain, Swati

Jana, Bimal

Jankowski, Mateusz

Janowski, Miroslaw

Jansen Van Vuren, Petrus

Januszkiewicz-Lewandowska, Danuta

Jaramillo Ortiz, José Manuel

Javelle, Emilie

Jeannot, Emilien
Jelinski, Murray

Jenkins, Frank

Jenner, Dominic C.

Jeong, Hye Won

Jerkic, Mirjana

Jerse, Ann E.

Jesudhasan, Palmy

Jeulin, Hélène

Jiménez-Jiménez, Felix Javier

Jin, Andrew

Jin, Jun-O

Jin, Liliang

Jin, Ling

Jin, Tengchuan

John, Jacob

John, Sinu P.

Johnson, Dylan M.

Johnson, Reed F.

Jones, Chris

Jones, Ian

Jones, Kenneth

Jones, Marjorie

Jones, Mark

Jones, Pauline

Jones, Rodney P.

Jose, Marco

Joshi, Kamal

Joubert, Annie

Jung, Klaus

Jung, Tae Sung

Jungersen, Gregers

Junior, Joao P. Araújo

Jurisic, Vladimir

Kabir, Russell

Kaddour, Hussein

Kaelber, Jason

Kagamu, Hiroshi

Kah, Janine

Kaliamurthi, Satyavani

Kammerer, Robert

Kampen, Jarl K.

Kanatani, Yasuhiro

Kanda, Tatsuo

Kandimalla, Ramesh

Kanekiyo, Masaru

Kang, Sang-Moo

Kanginakudru, Sriramana

Kanoi, Bernard

Kaplan, Shauna

Karaduta, Oleg

Karanika, Styliani 
Karlsson Hedestam, Gunilla B.

Karlsson, Erik

Karp, Jonathan D.

Karpenko, Larisa

Karsten, Christina B.

Kasprzak, Robert

Kaszowska, Marta

Kato, Fumihiro

Kato, Hideo

Katuchova, Jana

Kaul, Marcus

Kaushik, Radhey

Kawaguchi, Kazunori

Kawakami, Masanori

Kawano, Kouichiro

Kazimírová, Maria

Kazmi, Imran

Keele, John W.

Keeler, Shamus

Keller, Frieder

Kelly, Kevin M.

Kentaro, Yamada

Kenzaka, Tsuneaki

Kersten, Gideon

Kesari, Kavindra Kumar

Kesselring, Jürg

Keven, Joyal-Desmarais

Khalid, Hattaf

Khan, Krishnendu

Khan, Naeem

Khan, Rabia

Khanra, Nandish

Khatami, Ameneh

Khatun, Amina

Khubchandani, Jagdish

Kiara, Henry

Kido, Hiroshi

Kikuti, Mariana

Killian, Mary Lea

Kim, Gheun-Ho

Kim, Hae Won

Kim, Hei-sung

Kim, Hoon

Kim, Hye Kwon

Kim, In-Jeong

Kim, Jong

Kim, Jung Min

Kim, Ryong Nam

Kim, Sung-Kun (Sean)

Kipar, Anja

Kirchenbaum, Greg A.
Kirui, James

Kisa, Adnan

Kitagawa, Seiichi

Klampatsa, Astero

Klann, Jeffrey G.

Klaoudatos, Dimitrios

Klaumann, Francini

Kleinsorge, Thomas

Kluz, Tomasz

Ko, Hae-Jin

Koekemoer, Lizette

Kohler, James J.

Kokoszyński, Dariusz

Kolishetti, Nagesh

Komar, Nicholas

Komase, Katsuhiro

Kommineni, Nagavendra

Komori, Atsumasa

Koncz, Gábor

Kontsevaya, Irina

Köppen, Kristin

Kornilov, Sergey

Korzeń, Marcin

Kościelska-Kasprzak, Katarzyna

Koseva, Neli

Koshizuka, Tetsuo

Kosik, Ivan

Kosnik, Mitja

Kotozaki, Yuka

Kouji, Harada

Kouokam, Joseph Calvin

Koutselas, Ioannis

Kovalchuk, Alexander

Kovbasnjuk, Olga

Kowalska, Malgorzata

Krishnasamy, Gopinath

Kroeker, Andrea

Krug, Peter

Kuchar, Ernest Piotr

Kugelman, Jeffrey R.

Kuhlmann, F. Matthew

Kumagai, Makiko

Kumar, Amit

Kumar, Hirdesh

Kumar, Manish

Kumar, Raj

Kumar, Santosh

Kumar, Vikash

Kumararatne, Dinkantha

Kumaraswamy Naidu, Chitrala

Kumari, Sharda 
Kuryk, Lukasz

Kusumoto, Sojiro

Kwack, KyuBum

Kwakkel-van Erp, Hanneke

Kwan, Jennifer

L'homme, Laurent

La Porta, Raffaele

Laabei, Maisem

Labarère, José

Laboisse, Christian L.

Lada, Artem G.

Laher, Ismail

Lai, Alexander

Lai, Michael M.C.

Lal, Amos

Lambin, Philippe

Land, Kenneth C.

Landolt, Gabriele A.

Lang, Fengchao

Lange, Andrzej

Langermans, Jan A.M.

Laranjo, Marta

Larrous, Florence

Larsen, Kristian

Lasrado, Ninaad

Lau, Ho Yin Eric

Lau, Joseph Tak-fai

Lavender, Kerry

Lavoie, Kim L.

Law, John Lok Man

Le Doaré, Kirsty

Le Pendu, Jacques

Le, Hoang-Thanh

Leavenworth, Jianmei W.

Leclerc, Denis

Lecureux, Jonathan

Ledgerwood, Emily Desmet

Lee, Choongho

Lee, Howard

Lee, Hye-ra

Lee, Hyunju

Lee, Jihye

Lefebvre, Francois

Legaz, Isabel

Lei, Dylan

Leitão, Jorge H.

Leng, Anli

Leoncini, Lorenzo

León-Rubio, Jose María

Ley, Klaus

Leyson, Christina
Li, Ang

Li, Guangyu

Li, Jie

$\mathrm{Li}, \mathrm{Li}$

Li, Mei-Ling

Li, Shitao

Li, Xinbo

Li, Yonggang

Lian, Ie-Bin

Liang, Binhua

Liang, Xue-Hai

Liao, Yifei

Lichner, Zsuzsanna

Lichtenauer, Michael

Lichtenwalner, Anne

Lieber-Tenorio, Elisabeth

Liebman, Michael

Liechti, George W.

Ligat, Gaetan

Lim, Hui Yin

Lim, Siew Pheng

Lin, Chung-Ying

Lin, Chyongchiou J.

Lin, Haishu

Ling, Jiaxin

Lingwood, Daniel

Linial, Michal

Lio, Domenico

Lippke, Sonia

Liu, Haolin

Liu, Hejun

Liu, Huitao

Liu, Margaret

Liu, Saifei

Liu, Shih-Jen

Liutsko, Liudmila

Livaniou, Evangelia

Lo Presti, Elena

Lo, Shih-Yen

Loconsole, Daniela

Logue, James

Loh, Jacelyn

Lombardo, Giorgio

Lomonossoff, George P.

Long, Bradley

Long, Carrie Mae

Long, Chiau Ming

Long, Mengxian

López, Daniel

López-Siles, Mireia

Lorgelly, Paula 
Loubert, Linda

Lozano-Paniagua, David

Lu, Fengmin

Lucas, Sebastian

Lucas-Domínguez, Rut

Luciani, Mirella

Lukov, Georgi

Lundstrom, Kenneth

Lunel-Fabiani, Françoise

Lungu, Claudiu N.

Lüth, Stefan

Lv, Huibin

Lynge, Elsebeth

Macaluso, Giusi

Maccagno, Massimo

Macchi, Francesca

MacGregor, Casimir

Machida, Masaki

Maciver, Sutherland

MacRaild, Christopher

Madamsetty, Vijay Sagar

Madsen-Bouterse, Sally A.

Maeda, Noriaki

Maekawa, Shun

Magariños Ferro, Beatriz

Maglie, Roberto

Magnavita, Nicola

Magurano, Fabio

Mahmoud, Ayman

Maietti, Elisa

Maisey, Kevin

Majer, Anna

Malchiodi, Emilio L.

Maleki Vareki, Saman

Malinauskas, Romualdas

Malleret, Benoit

Mallo, Natalia

Maloberti, Alessandro

Mamidi, Murali

Manam, Srikanth

Mancianti, Francesca

Mandel, Micha

Mandilara, Georgia

Manfredi, Marcello

Mangsbo, Sara M.

Manning, Jessica

Manzoli, Lamberto

Maraolo, Alberto Enrico

Marasca, Claudio

Marascio, Nadia

Marć, Małgorzata Anna
Marchesi, Alessandra

Marchesi, Federico

Marcilla, Antonio

Marco, Dadda

Marenzoni, Maria Luisa

Marguello, Miguel Santibañez

Marimon, Jose Maria

Marinelli, Enrico

Marinos, Georgios

Marion, Jason W.

Marotta, Claudia

Marsilio, Fulvio

Marson, Piero

Martín, Verónica

Martinelli, Chiara

Martin-Galiano, Antonio J.

Martín-García, Elena

Martinho, José Pedro

Martins, Andrew

Martins, Jorge N.R.

Marzi, Andrea

Marzuillo, Carolina

Mascarenhas, Maya

Masiulis, Marius

Maslow, Joel N.

Masoller, Narcís

Masresha, Balcha

Mastalerz-Migas, Agnieszka

Mastino, Antonio

Mastrangelo, Peter

Matarese, Giovanni

Mateos-Hernández, Lourdes

Mathieu, Cyrille

Mathioudakis, Alexander G.

Matsuo, Eiko

Matsuo, Kazuhiko

Mattei, Fabrizio

Mattiuzzo, Giada

Mattner, Jochen

Mattoscio, Domenico

Matveeva, Olga

Maurin, Max

Maurya, Shailendra

Mayner, Lidia

Mazor, Ohad

Mazurov, Dmitriy V.

McAndrew, Siobhan

McBride, John

McElroy, Jane A.

McIntosh, E. David G.

McKinstry, Karl Kai 
McLaren, James E.

McLucas, Bruce

McVey, David

Medina, Gisselle N.

Mei, Kuo-Ching

Mellinghoff, Sibylle

Ménard, Hervé

Mendonça-Lima, Leila

Mereu, Alessandra

Merino, Jose Joaquin

Merritt, Rowena

Mertig, Angela

Mesch, Gustavo

Mesquita, João

Mestres, Conxita

Metovic, Jasna

Meurens, François

Meyer, Sascha

Meyerhans, Andreas

Meysamie, Alipasha

Mhatre, Siddhita

Miao, Qiuhong

Michos, Athanasios G.

Migliore, Sergio

Migliorini, Paola

Milani, Gregorio P.

Milardi, Danilo

Milavetz, Barry

Milella, Michele

Miller, Elizabeth

Mingorance, Jesús

Miniero, Daniela Valeria

Minutolo, Antonella

Mirza, Imran

Mishra, Aarti

Mishra, Manish

Mishra, Priyanka

Mishra, Sanket

Mishto, Michele

Mitov, Mihail

Mitra, Amal K.

Mitsios, Alexandros

Miyagi, Etsuko

Moccia, Marcello

Modenese, Alberto

Modhiran, Naphak

Mohammed, Altaf

Mohsen, Mona

Moiré, Nathalie

Mojumdar, Kamalika

Molarius, Anu
Molenaar, Remco J.

Momotani, Eiichi

Mongin, Denis

Montalti, Marco

Montalto, Luigi

Monteiro, Sílvia

Montes, Nuria

Montgomery, Courtney Gray

Montoya-Alonso, José Alberto

Moore, Justin Xavier

Mooyottu, Shankumar

Morales Suárez-Varela, María M.

Morales, María

Mora-Montes, Hector

Morciano, Giampaolo

Morea, Donato

Moreira, Ana Maria Da Palma

Moreno, Esther

Moretti, Sonia

Mori, Masaaki

Morpurgo, Margherita

Morrison, Ivan

Mortara, Lorenzo

Mosconi, Giovanni

Motamed, Cyrus

Mouchtouri, Varvara A.

Mould-Quevedo, Joaquin F.

Mousa, Jarrod J.

Moutschen, Michel

Moya Higueras, Jorge

Msallam, Rasha A.

$\mathrm{Mu}$, Qinghui

Mueller, Judith E.

Mukai, Hidehito

Mukai, Tomoyuki

Mukherjee, Anupam

Mukherjee, Jean

Mukherjee, Soumyadeep

Mukiibi, Robert

Mukundan, Santhosh

Mulcahy, Grace M.

Mulder, Bob

Muller, Mandy

Müller, Martin

Müller-Trutwin, Michaela

Múñez, Elena

Muniraju, Murali

Muñoz-Barroso, Isabel

Mura, Cameron

Murale, Dhiraj P.

Murata, Shiro 
Murdaca, Giuseppe

Murmura, Federica

Murphy, Denis

Murugan, Natarajan Arul

Murugan, Rajagopal

Murugan, Sengottuvelan

Musalgaonkar, Sharmishtha

Mutinelli, Franco

Mutz, Pascal

Muxel, Sandra Marcia

Myc, Andrzej

Mytych, Jennifer

Nabavi, Seyed Massood

Nacher, Mathieu

Naguib, Mahmoud

Nair, Smita

Nakamizo, Tomoki

Nakamura, Kyohei

Nalos, Marek

Nam, Taewoo

Napoli, Christian

Nardini, Roberto

Naruse, Hiroyuki

Narzisi, Antonio

Nasar, Farooq

Nath, Niharika

Naylor, Andrew

Nazki, Salik

Nde, Pius N.

Neely, Melody N.

Neerukonda, Naga Venkata Sabarinath

Negri, Donatella

Nekhai, Sergei

Nellimarla, Srinivas

Neter, Efrat

Netesov, Sergey

Neuman, Benjamin

Newman, John F.

$\mathrm{Ng}$, Qin Xiang

Nghia, Truong Phuoc

Nghiem, Son

Ngunjiri, John

Nguyen, Duc-Hiep

Ní Cheallaigh, Clíona

Nicoletti, Loredana

Nicoli, Francesco

Nikbakht Nasrabadi, Alireza

Nikolaou, Elissavet

Nikolich, Mikeljon

Nishizono, Akira

Nissapatorn, Veeranoot
Nistal-Villán, Estanislao

Nitta, Takayuki

Nogales, Aitor

Noh, Jae Myoung

Nomizo, Takashi

Novick, Daniela

Novikov, Alexander

Novo-Veleiro, Ignacio

Nowak, Glen

Ntanasis-Stathopoulos, Ioannis

Nunez, Alejandro

O'Connor, Daniel

O'Reilly, Mary

Odone, Anna

Oey, Melanie

Ohkuri, Takayuki

Okamoto, Shigefumi

Okazaki, Katsunori

Okumura, Nobuaki

Oliveira, Guilherme

Oliver, JoAnne

Olivetta, Eleonora

Olsen, Daniel

Onorato, Lorenzo

Onyilagha, Chukwunonso

Opata, Michael

Ord, Keith

Ortega, Joseph Thomas

Ortega, Miguel A.

Ortega, Nieves

Orusa, Riccardo

Osuna, Eduardo

Otani, Naruhito

$\mathrm{Ou}$, Chun-Quan

Oyekale, Abayomi

Ozaki, Shozo

Ozdarendeli, Aykut

Pakpour, Amir Haji Agha

Pal, Dhiman

Palaiodimos, Leonidas

Palaniswamy, Saranya

Palazón-Bru, Antonio

Palladino, Raffaele

Palmberger, Dieter

Palmisano, Alida

Palumbo, Marco

Pan, Xiaoling

Pandey, Rajan

Panella, Massimiliano

Pang, Iris

Papadopoulos, Georgios 
Papandreou, Nikolaos

Papazisis, Georgios

Paran, Nir

Parente, Paola

Parisini, Emilio

Park, Eun Jeong

Park, Jung-Eun

Parronchi, Paola

Parvate, Amar

Pascutti, Maria Fernanda

Patching, Simon G.

Patel, Sonal

Patrianakos, Alexandros

Pavelko, Kevin D.

Pavone, Piero

Paweska, Janusz

Peabody, David

Pechous, Roger

Peghin, Maddalena

Pei, Yonggang

Peixe, Paula

Pejchal, Jaroslav

Pellegrinelli, Laura

Pépin, Michel

Perch, Michael

Perche, Federico

Perdomo, Jose

Pereira, Maria

Perez, Francisco

Perez, Javier J.

Pérez-Bermejo, Marcelino

Perrella, Alessandro

Perricone, Carlo

Pessela João, Benevides

Petraityte-Burneikiene, Rasa

Petrillo, Marco

Petrini, Stefano

Pezzoni, Giulia

Pfaller, Christian

Pflughoeft, Kathryn

Phanse, Yashdeep

Phogat, Sanjay

Piantino Ferreira, Antonio José

Piedra, Pedro A.

Pierannunzio, Daniela

Pileggi, Claudia

Pimpinelli, Fulvia

Pinacchio, Claudia

Pincus, Matthew R.

Pincus, Seth

Pires, Carla
Piscopo, Marina

Piskin, Senol

Piu, Saha

Plans-Rubió, Pedro

Plant, Ewa

Pokorski, Mieczyslaw

Poluzzi, Elisabetta

Poma, Adolfo

Pomara, Cristoforo

Pomastowski, Paweł P.

Poniedzialek, Barbara

Poo, Haryoung

Poole, Brian D.

Popa, Mircea Ioan

Pouliakis, Abraham

Poveda, Cristina

Pozzi, Cecilia

Prakas, Petras

Prasad, Abhishek

Pratesi, Federico

Price, Patricia

Prokop, Jeremy W.

Prospero, Emilia

Protopopescu, Camelia

Prüller, Florian

Psarra, Katerina

Puig-Barberà, Juan

Pulido, Olga

Punetha, Ankita

Purvis, Rachel

Pustylnikov, Sergey

Pyo, Hyun Mi

Pyzik, Michal

Qu, Bingqian

Quiñones-Mateu, Miguel E.

Rachiotis, George

Radcliff, Fiona Jane

Radin, Massimo

Raeven, René

Rafaniello, Concetta

Raftery, Martin

Ragan, Izabela

Rahimi, Siavash

Rajaiya, Jaya

Rajkumar Singh, Kalra

Rajnik, Michael

Rakotondrafara, Aurelie

Ramadori, Giuliano

Ramasamy, Ranjan

Ramatchandirin, Balamurugan

Ramchandani, Divya 
Ramírez-Paredes, Jose Gustavo

Ramírez-Toloza, Galia A.

Ramos-Morcillo, Antonio Jesús

Rani Banik, Gouri

Ranzato, Elia

Rao, Guodong

Rao, Praveen P.N.

Rapi, Stefano

Rapone, Biagio

Ratajczak, Magdalena

Rather, Irfan

Rauf, Mohd

Raundhal, Mahesh

Rava, Marta

Ravindra, P. Veeranna

Ray, Samriddha

Raze, Dominique

Razzuoli, Elisabetta

Real-Fernández, Feliciana

Reche, Pedro

Reece, Jeanette

Regla-Nava, Jose A.

Regmi, Krishna

Reid, Scott

Reis, Ana

Remmerswaal, Ester B.M.

Renu, Sankar

Reyes-del Valle, Jorge

Reynolds, Matthew R.

Riccò, Matteo

Richards, Stephen J.

Richardson, Jennifer

Richner, Justin M.

Rigby, Michael

Rinnerthaler, Mark

Riquelme, Erick

Rissanen, Ilona

Riva, Giuseppe

Rizzo, Alessandro

Roach, Jared C.

Robinson, Sally R.

Robles-Marhuenda, Angel

Roby, Justin A.

Roccetti, Marco

Rocha Faustino, Ana Isabel

Rodrigues, Andreia C.M.

Rodríguez Ortega, Manuel José

Rodríguez, Fernando

Roh, Changhyun

Rohaim, Mohammed

Rojo, Maria Angeles
Roldão, António

Rolla, Giovanni

Rolla, Simona

Romano, Ferdinando

Romano, Nicla

Rong, Chan Kuan

Roni, Monzurul

Ronsard, Larance

Root-Bernstein, Robert

Rosenthal, Ken

Rossetti, Carlos A.

Roubalova, Katerina

Rouleau, Matthieu

Routhu, Nanda Kishore

Rouzine, Igor

Rovin, Richard A.

Rowland-Jones, Sarah

Roy, Indrani

Rübsamen, Nicole

Ruggiero, Alessandra

Ruggiero, Giuseppina

Ruiz, Maurici

Rukambile, Elpidius J.

Runtuwene, Lucky Ronald

Ruotsalainen, Janne

Russell, Alistair

Russo, Momtchilo

Ruysschaert, Jean Marie

Ružauskas, Modestas

Rybka, Jakub

Ryter, Kendal

Sabbatucci, Michela

Sabeta, Claude

Sabir, Hemmen

Sachithanandham, Jaiprasath

Sagnelli, Caterina

Sahu, Kamal Kant

Sahu, Rajnish

Said, Elias A.

Sakač, Nikola

Sakellari, Evanthia

Saksena, Nitin

Saláková, Martina

Salali, Gul Deniz

Salerno, Laura

Salio, Mariolina

Sallam, Malik

Salman, Mootaz

Salpini, Romina

Salvato, Maria

Salzman, Mark B. 
Samir, Parimal

Samy, Abdallah

Sánchez Crespo, Mariano

Sanchez, David Jesse

Sandonà, Dorianna

Sándor, Attila D.

Sannella, Alessandra

Santacroce, Luigi

Santarem, Nuno

Santos, Adriana O.

Santos, Jefferson

Sardu, Claudia

Sarkar, Amrita

Sarker, Subir

Sarría Santamera, Antonio

Sasaki, Hiraku

Sato, Hiroshi

Satoshi Kashiwagi, Satoshi

Sattentau, Quentin

Sattler, David N.

Saunders, John

Sauter-Louis, Carola

Saviano, Angela

Savoia, Elena

Sawanyawisuth, Kittisak

Saxena, Pooja

Sayed, Ibrahim M.

Sayedahmed, Ekramy

Scapigliati, Giuseppe

Scarpa, Fabio

Schaefer, Alexandra

Schetters, Sjoerd T.T.

Schirhagl, Romana

Schirtzinger, Erin E.

Schlecht-Louf, Géraldine

Schmaljohn, Alan

Schmidt, Martin

Schmitt, Anthony

Schmoll, Dieter

Schoenhagen, Paul

Schulte, Bianca

Schwarz, Anke

Schwarz, Lukas

Schwarzinger, Michaël

Schweizer, Matthias

Schwermer, Heinzpeter

Schwetz, Zollner

Scioscia, Crescenzio

Scomparin, Anna

Scorpio, Diana

Scott, Daniel
Scott, David

Scotti, Marcus

Seals, Brenda F.

Sebastian, Robin

Sebina, Ismail

Seed, Sheila

Seike, Masahiro

Selim, Abdelfattah M.

Selvaraj, Arokiyaraj

Selvaraj, Periasamy

Senna, Gianenrico

Seo, Giwan

Seo, Ho Seong

Seo, Sang Heui

Seow, Jeffrey

Sessa, Francesco

Seth, Anushree

Sethi, Gautam

Sethi, Manveen

Sevdalis, Nick

Sharma, Piyush

Sharma, Prashant

Sharma, Shweta

Sharonov, George Vladimirovich

Shaukat, Aftab

Shaw, Andrew

Sheikh, Abu Baker

Sheikh, Imlak

Shelley, Mack

Shelton, Holly

Shemirani, Amir

Shen, Yang

Shenoy, Anukul T.

Sherwani, Mohammad Asif

Shestopalov, Alexander M.

Sheu, Jim

Shimek, Jo Anna

Shimodaira, Shigetaka

Shin, Crystal S.

Shin, Hyoung-shik

Sibley, David

Siggins, Robert W.

Signori, Alessio

Sikalidis, Angelos

Siltz, Lauren A.

Silva, Abigail

Silva, Ediane

Silva, Marcelo Sousa

Silva, Severino Jefferson Ribeiro

Silva-Júnior, Abelardo

Silvestri, Giovannino 
Simecka, Jerry W.

Simões, João Carlos Caetano

Simões, Margarida Pires

Singanalur, Nagendrakumar Balasubramanian Singh, Amit K.

Singh, Brajesh Kumar

Singh, Kamalendra

Singh, Mahima

Singh, Sandeep

Singh, Sarbjit

Sinigaglia, Alessandro

Sioofy-Khojine, Amir-Babak

Siristatidis, Charalampos

Skinner, Jack

Skirrow, Helen

Skwarczynski, Mariusz

Slonchak, Andrii

Śmiałek, Marcin

Smith, Richard

Smith, Todd G.

Smola, Jiri

Smulski, Sebastian

Sneddon, Jennifer

Sochocka, Marta

Sokoloski, Kevin

Solís García Del Pozo, Julián

Sominskaya, Irina

Song, Shuang

Sonnino, Giorgio

Soreq, Lilach

Sousa, Angela

Spann, Kirsten

Speijer, Dave

Spellerberg, Barbara

Spencer, Juliet V.

Sperone, Emilio

Spinu, Marina

Spiro, David J.

Sprenger, Kayla

Spurgeon, Megan

Sridhar, Siddharth

St.Cyr, John A.

Staats, Herman F.

Stanberry, Lawrence R.

Stapleford, Kenneth

Stasi, Alessandra

Stauber, Roland

Steele, Duncan

Stefanini, Lucia

Stefanoff, Pawel

Steffen, David
Steinberg, Julia R.

Steinborn, Michael

Steiner, Margarete

Steinhagen, Dieter

Steinke, Douglas

Stephens, Edward

Sternberg, Robert

Stoecker, Charles

Stoiber, Heribert

Stone, Merlin

Streck, André Felipe

Strohfus, Pamela K.

Subbiah, Jeeva

Sue, Shih-Che

Sugawara, Norio

Sugiura, Katsuaki

Suliman, Hagir B.

Sullivan, John Stephen

Summerfield, Artur

Sun, Chenyu

Sun, Xiangjie

Superti, Fabiana

Suryadevara, Naveenchandra

Suschak, John J.

Swain, Jaya Kumari

Szabó, László

Szymaniec-Mlicka, Karolina

Szymczak, Aleksandra

Tafforeau, Lionel

Tafuri, Silvio

Tagliavia, Marcello

Taitel, Michael

Tajima, Motoshi

Takahashi-Omoe, Hiromi

Takayama-Ito, Mutsuyo

Takeda, Makoto

Talakić, Emina

Tamayo, Esther

Tana, Claudio

Tandukar, Sarmila

Tang, Jianming

Tanno, Luciana Kase

Tarlinton, Rachael

Tárraga Mínguez, Raúl

Tārs, Kaspars

Tartey, Sarang

Tavares, Joana

Taylor, Andrew W.

Tazzari, Marcella

Tellier, Michael

Terán, Enrique 
Thacker, Naveen

Thannesberger, Jakob

Theron, Jacques

Thomas, Roger E.

Thompson, Andrew J.

Thompson, Craig

Thompson, Dorothea K.

Tinker, Juliette

Tittarelli, Andrea

Tiwari, Pooja

Todorov, Svetoslav

Tognetto, Alessia

Toietta, Gabriele

Tomaszewska, Ewa

Tonelli, Michele

Toniolo, Antonio Q.

Toranzo, Alicia E.

Torner, Nuria

Toronto, Coleen E.

Torres-Fernández, Orlando

Torres-Macho, Juan

Torri, Emanuele

Toth, Istvan

Toyos, Juan R. De Los

Trafimow, David

Trama, Francesco

Trejbalova, Katerina

Trevisan, Andrea

Tripathi, Ashutosh

Tripathi, Jitendra

Tripathi, Jitendra Kumar

Trollfors, Birger

Trujillo, Jessie

Truman, Benedict I.

Trus, Ivan

Tsai, Ming-Horng

Tsergouli, Katerina

Tsikouras, Panagiotis

Tsioufis, Konstantinos

Tung, Tao-Hsin

Turbow, David

Turillazzi, Emanuela

Tyagi, Mudit

Ucciferri, Claudio

Uddin, Md Jamal

Uetrecht, Charlotte

Ulbert, Sebastian

Ullah, Md Ashik

Ulu, Arzu

Urban, Britta

Ussowicz, Marek
Uthaman, Saji

Uthman, Olalekan

Vabulas, R. Martin

Vaismoradi, Mojtaba

Valasoulis, George

Valicherla, Guru Raghavendra

Valle, Manuel Rodriguez

Van Der Vossen, Jos

Van Gisbergen, Marike W.

Van Gorp, E.C.M. (Eric)

Van Hemert, Martijn

Van Keulen, Britt J.

Van Wagoner, Nicholas J.

Varadarajan, Janani

Varani, Luca

Vardasca, Ricardo

Varol, Serkan

Varsani, Arvind

Vasile, Francesca

Vasilyev, Kirill

Vázquez-Domínguez, Evaristo

Veiga, Ana Gorini Da

Velazquez-Salinas, Lauro

Velin, Dominique

Veltri, Giuseppe Alessandro

Venancio, Emerson José

Verros, George D.

Villalba-Montoro, Rafael

Vimercati, Luigi

Virtaneva, Kimmo

Vistoli, Giulio

Visvizi, Anna

Visweswaran, Ganesh Ram

Vitale, Chiara

Viviani, Simonetta

Vogiatzaki, Chrysanthi

Voigt, Emily A.

Von Horsten, Hans Henning

Vrentas, Catherine E.

Vuillefroy De Silly, Romain

Wada, Koji

Walduck, Anna K.

Wang, Fun-In

Wang, Guozheng

Wang, Hsin-Yao

Wang, Jiun-Lin

Wang, Jun

Wang, Junfeng

Wang, Kai

Wang, Minghang

Wang, Robert Yung-Liang 
Wannemuehler, Michael J.

Want, Muzamil Yaqub

Ward, Brian M.

Wasniewski, Marine

Waterman, Paige E.

Waye, Mary M.Y.

Wei, Hongping

Wei, Jin

Wei, Ruifang

Weina, Peter J.

Weingartner, Magdalena

Weinrick, Brian C.

Weiss, Christel

Wen, Conghua

Werner, Enrique

Wettstein, Martin

White, Jacquie

Wieckowski, Sébastien

Williams, Edgar Mark

Williams, Jessica A.

Williams, Susan

Williamson, Ethel Diane

Wilm, Matthias

Wilson, Alphus D.

Wilson, Heather

Wilson, Jo Leanne

Wilusz, Jeff

Wissmath, Bartholomäus

Wohlleber, Dirk

Wölfel, Roman

Wolfraim, Larry

Wolfrum, Nina

Wong, Chi Chun

Woods, Jon P.

Woroniecka, Karolina

Wozniak-Knopp, Gordana

Woźniakowski, Grzegorz

$\mathrm{Wu}$, Hannah

Wu, Jack H.Z.

$\mathrm{Wu}$, Jian

Wu, Jianxuan

$\mathrm{Wu}$, Mei X.

Wubishet, Befikadu

Xia, Shuai

Xie, Hang

Xie, Jian

$X u$, Zhi Ping

Xue, Guangai

Xue, Min
Yadav, Dharmendra K.

Yager, Eric

Yamamoto, Kei

Yang, Ming

Yaparla, Amulya

Ye, Yaozong

Yen, Cheng-Fang

Yin, Li

Yoon, Kyungjae-andrew

York, Ian A.

Yoshida, Noriaki

Yoshio, Sachiyo

Yost, Christian C.

Youn, Bo-Young

Younes, Husam

Young, Sean G.

Yousefi, Morteza

Yuan, Lijuan

Yuan, Meng

Zahar, Jean Ralph

Zahm, Christopher D.

Zahn, Roland C.

Zamperin, Gianpiero

Zapata, Juan Carlos

Zappulla, David C.

Zavras, Dimitris

Zefferino, Roberto

Zeichner, Steven L.

Zelger, Bernhard

Zella, Davide

Zeng, Minxiang

Zeynaloo, Elnaz

Zhang, Duo

Zhang, Jianqiang

Zhang, Kun

Zhang, Lizhou

Zhang, Shuo

Zhao, Linbo

Zhou, Dongming

Zhou, Fan

Zhou, Lang

Zidovec Lepej, Snjezana

Zilliox, Michael J.

Zona, Stefano

Zost, Seth

Zou, Keyuan

Zumbrun, Elizabeth

Zyśko, Dorota 